\title{
Front Matter: Volume 10354
}

, "Front Matter: Volume 10354," Proc. SPIE 10354, Nanoengineering: Fabrication, Properties, Optics, and Devices XIV, 1035401 (25 September 2017); doi: 10.1117/12.2296967

SPIE Event: SPIE Nanoscience + Engineering, 2017, San Diego, California, United SPIE. States 


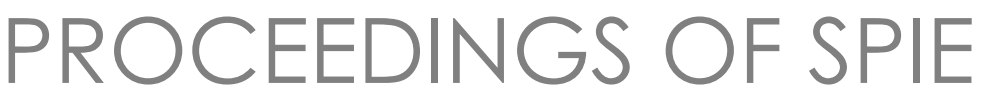

\title{
Nanoengineering: Fabrication, Properties, Optics, and Devices XIV
}

\author{
Eva M. Campo \\ Elizabeth A. Dobisz \\ Louay A. Eldada \\ Editors
}

9-10 August 2017

San Diego, California, United States

Sponsored and Published by

SPIE 
The papers in this volume were part of the technical conference cited on the cover and title page. Papers were selected and subject to review by the editors and conference program committee. Some conference presentations may not be available for publication. Additional papers and presentation recordings may be available online in the SPIE Digital Library at SPIEDigitalLibrary.org.

The papers reflect the work and thoughts of the authors and are published herein as submitted. The publisher is not responsible for the validity of the information or for any outcomes resulting from reliance thereon.

Please use the following format to cite material from these proceedings:

Author(s), "Title of Paper," in Nanoengineering: Fabrication, Properties, Optics, and Devices XIV, edited by Eva M. Campo, Elizabeth A. Dobisz, Louay A. Eldada, Proceedings of SPIE Vol. 10354 (SPIE, Bellingham, WA, 2017) Seven-digit Article CID Number.

ISSN: 0277-786X

ISSN: 1996-756X (electronic)

ISBN: 9781510611658

ISBN: 9781510611665 (electronic)

Published by

SPIE

P.O. Box 10, Bellingham, Washington 98227-0010 USA

Telephone +1 3606763290 (Pacific Time) · Fax +1360647 1445

SPIE.org

Copyright @ 2017, Society of Photo-Optical Instrumentation Engineers.

Copying of material in this book for internal or personal use, or for the internal or personal use of specific clients, beyond the fair use provisions granted by the U.S. Copyright Law is authorized by SPIE subject to payment of copying fees. The Transactional Reporting Service base fee for this volume is $\$ 18.00$ per article (or portion thereof), which should be paid directly to the Copyright Clearance Center (CCC), 222 Rosewood Drive, Danvers, MA 01923. Payment may also be made electronically through CCC Online at copyright.com. Other copying for republication, resale, advertising or promotion, or any form of systematic or multiple reproduction of any material in this book is prohibited except with permission in writing from the publisher. The CCC fee code is 0277$786 \mathrm{X} / 17 / \$ 18.00$.

Printed in the United States of America.

Publication of record for individual papers is online in the SPIE Digital Library.

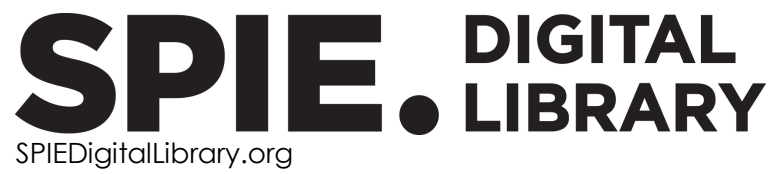

Paper Numbering: Proceedings of SPIE follow an e-First publication model. A unique citation identifier (CID) number is assigned to each article at the time of publication. Utilization of CIDs allows articles to be fully citable as soon as they are published online, and connects the same identifier to all online and print versions of the publication. SPIE uses a seven-digit CID article numbering system structured as follows:

- The first five digits correspond to the SPIE volume number.

- The last two digits indicate publication order within the volume using a Base 36 numbering system employing both numerals and letters. These two-number sets start with 00, 01, 02, 03, 04, 05, 06, 07, 08, 09, 0A, OB ... 0Z, followed by 10-1Z, 20-2Z, etc. The CID Number appears on each page of the manuscript. 


\title{
Contents
}

\author{
vii Authors \\ ix Conference Committee
}

\section{SESSION $1 \quad$ PHOTONIC DEVICES}

1035403 Large-scale fabrication of LP-CVD Si3 $\mathrm{N}_{4}$ photonic crystal structures as freestanding reflectors with $1 \mathrm{~mm}$ aperture for Fabry-Pérot interferometers [10354-1]

1035404 Actuated polymer based dielectric mirror for visual spectral range applications [10354-2]

1035406 Limited-size guided-mode resonance filters under focused beams [10354-4]

1035407 Effects of coupling configuration on resonance excitation in a slotted photonic crystal nanobeam [10354-5]

1035408 Steering and filtering white light with resonant waveguide gratings [10354-6]

\section{SESSION 2 INNOVATIVE DEVICES}

10354 OA Contactless capacitive adiabatic logic [10354-8]

10354 OC Development of sensitive holographic devices for physiological metal ion detection [10354-10]

\section{SESSION 3 NANOPATTERNING}

$10354 \mathrm{OE}$ Scalable maskless patterning of nanostructures using high-speed scanning probe arrays [10354-13]

$10354 \mathrm{OF}$ Impact of processing and growth conditions on the site-catalyzed patterned growth of GaAs nanowires by molecular beam epitaxy [10354-14]

10354 OG Innovative patterning method for modifying few-layer $\operatorname{MoS}_{2}$ device geometries [10354-15]

\section{SESSION 4 ENGINEERED MATERIALS}

$10354 \mathrm{OH} \quad$ Update on bio-refining and nanocellulose composite materials manufacturing (Invited Paper) [10354-16]

10354 Ol All dielectric metasurface nano-fabrication based on $\mathrm{TiO}_{2}$ phase shifters [10354-17] 
10354 0J A nanostructure based on metasurfaces for optical interconnects [10354-18]

$10354 \mathrm{OL}$ Handling and assembling of low-density foam structures fabricated by two-photon polymerization [10354-20]

\section{SESSION 5 CARBON NANOTUBES AND LOW-DIMENSIONAL MATERIALS}

1035400 Low contact resistance of the MWCNTs ohmic contact to p-GaN and its application for high power LED [10354-23]

10354 OP High aspect ratio CNT structures produced by energetic ion bombardment [10354-24]

$10354 \mathrm{OQ}$ Time-domain finite-difference based analysis of induced crosstalk in multiwall carbon nanotube interconnects [10354-25]

10354 OS Surface plasmon resonance based electro-optic measurement of SBN thin films [10354-27]

SESSION $6 \quad$ LIGHT TRAPPING AND LIGHT GUIDING

$10354 \mathrm{OU} \quad$ Chromatic photo-thermal actuators based on 2H-MoS 2 based nanocomposites [10354-29]

10354 OV Luminescence studies of laser MBE grown GaN on ZnO nanostructures [10354-30]

\section{SESSION 7 NANO-OPTIC DEVICES AND OPTIC ENGINEERING}

10354 OY Subwavelength 2D segmented waveguide and light coupling optimization by evolutionary algorithms [10354-33]

1035410 Design of binary patterns for speckle reduction in holographic display with compressive sensing and direct-binary search algorithm [10354-36]

1035411 Vanadium dioxide switchable components based on wiregrids for mid-infrared applications [10354-43]

\section{POSTER SESSION}

1035412 Design and calibration of a nano dimensional standard [10354-37]

1035414 Extreme-ultraviolet and electron beam lithography processing using water developable resist material [10354-39]

1035417 Modularized and water-cooled photo-catalyst cleaning devices for aquaponics based on ultraviolet light-emitting diodes [10354-42]

10354 1B High resolution patterning of ultraviolet cross-linked resins using gas permeable mold derived from cellulose in nanoimprint lithography [10354-47] 
10354 1D The challenge of screen printed Ag metallization on nano-scale poly-silicon passivated contacts for silicon solar cells [10354-49]

$10354 \mathrm{lE} \quad$ Temperature dependency of mechanical properties for crystalline cellulose added to silicone elastomer [10354-50]

$10354 \mathrm{IF}$ Temperature dependence of viscoelasticity of crystalline cellulose with different molecular weights added to silicone elastomer [10354-51]

$103541 \mathrm{~J} \quad$ Mechanical performance of SiC based MEMS capacitive microphone for ultrasonic detection in harsh environment [10354-55]

$103541 \mathrm{~K} \quad \mathrm{MoS}_{2}$ thin films prepared by sulfurization [10354-56]

$10354 \mathrm{IM}$ The diffraction patterns of the output light from the tapered fiber tips [10354-58]

$103541 \mathrm{~N}$ Complementary methods of study for $\mathrm{Zr}_{1-x} \mathrm{Ce}_{\mathrm{x}} \mathrm{O}_{2}$ compounds for applications in medical prosthesis [10354-59]

10354 IP Designer metamaterials using graphene for integrated nano-photonic applications [10354-61]

$103541 Q \quad$ Organic-inorganic hybrid resist materials in advanced lithography [10354-62]

10354 IT N-state random switching based on quantum tunnelling [10354-65]

$103541 \mathrm{U}$ Three-dimensional printing and deformation behavior of low-density target structures by two-photon polymerization [10354-66] 
Proc. of SPIE Vol. 10354 1035401-6

Downloaded From: https://www.spiedigitallibrary.org/conference-proceedings-of-spie on 26 Apr 2023 Terms of Use: https://www.spiedigitallibrary.org/terms-of-use 


\section{Authors}

Numbers in the index correspond to the last two digits of the seven-digit citation identifier (CID) article numbering system used in Proceedings of SPIE. The first five digits reflect the volume number. Base 36 numbering is employed for the last two digits and indicates the order of articles within the volume. Numbers start with 00, 01, 02, 03, 04, 05, 06, 07, 08, 09, 0A, 0B...0Z, followed by 10-1Z, 20-2Z, etc.

\author{
Afzal, Francis, 07 \\ Akella, Meghana, OE \\ Astbury, Benjamin, $1 T$ \\ Basset, Guillaume, 08 \\ Basset, Philippe, OA \\ Becht, Gregory, 1D \\ Bernardo-Gavito, Ramón, 0G, $1 T$ \\ Bierret, Antoine, 06 \\ Black, Andrés, 0G \\ Bruma, Alina, 1N \\ Burkhovetsky, Valerii $V_{\text {., }}$ iN \\ Cahayag, R., OL \\ Campbell, John H., $1 \mathrm{U}$ \\ Casado, Santiago, OG \\ Cassidy, John, OC \\ Chen, Chen, OE \\ Chen, Jiun-An, $1 M$ \\ Chen, Xin, 12 \\ Chen, Zi-Yu, 17 \\ Chou, Yu-Yang, 17 \\ Chromik, Š., $1 \mathrm{~K}$ \\ Craus, Mihail-Liviu, $1 \mathrm{~N}$ \\ Dewan, Sheetal, OV \\ Ding, Guoqing, 12 \\ Dobročka, E., $1 \mathrm{~K}$ \\ Dourado-Sisnando, A., OY \\ Du, Zhidong, OE \\ Dubos, Pierre-Antoine, $1 \mathrm{~N}$ \\ F. Vieira, L., OY \\ Fanet, Hervé, OA \\ Galisultanov, Ayrat, OA \\ Gallinet, Benjamin, 08 \\ Granados, Daniel, OG \\ Gu, Huarong, OJ \\ Guo, Pengfei, 11 \\ Gupta, Surbhi, OS \\ Gupta, Vinay, OS, OV \\ Haïdar, Riad, 06 \\ Hamzah, A. A., $1 \mathrm{~J}$ \\ Han, Tao, 12 \\ Hanabata, Makoto, 1B, 1E, IF, 1Q \\ Helke, Christian, 03 \\ Hiller, Karla, 03 \\ Hoerteis, Matthias, ID \\ Huang, Yi-Bo, 17 \\ Hulman, M., IK \\ Hutár, P., $1 \mathrm{~K}$ \\ Hwang, Do Kyung, 10 \\ Iyer, Shanthi, OF
}

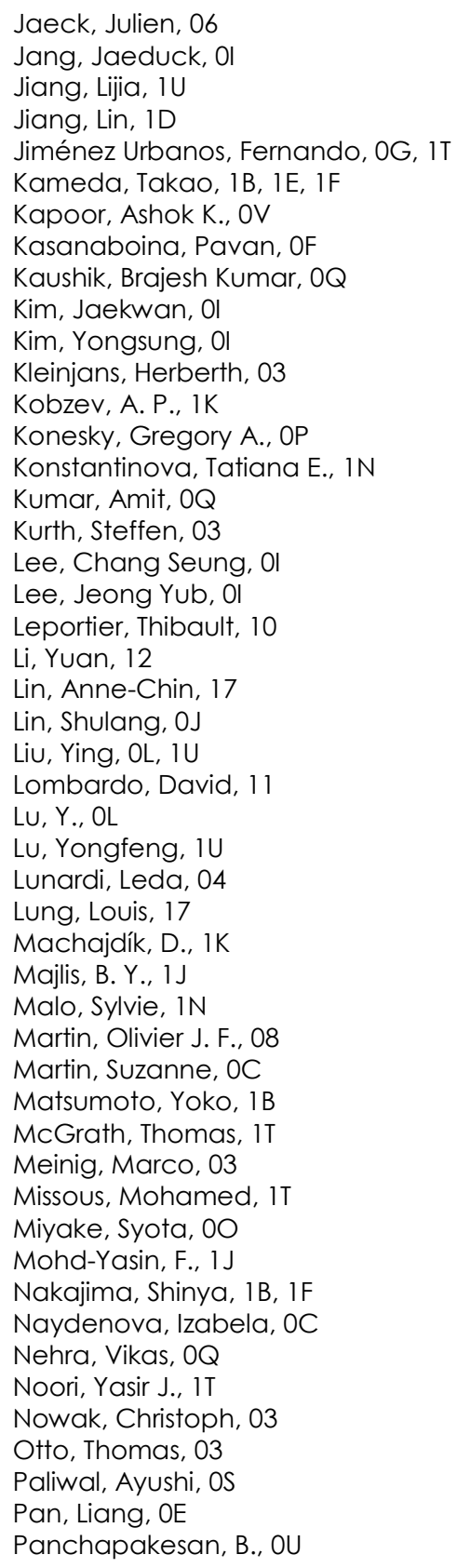


Pardo, Fabrice, 06

Park, Min-Chul, 10

Pelouard, Jean-Luc, 06

Perrin, Yann, OA

Petta, Nicole, OL, IU

Pillonnet, Gaël, OA

Postek, Michael T., $\mathrm{OH}$

Poster, Dianne L., $\mathrm{OH}$

Quaranta, Giorgio, 08

R. Osorio, Manuel, OG

Rahneshin, $V_{\text {., OU }}$

Reuter, Danny, 03

Roberts, Jonathan, $1 \mathrm{~T}$

Rodríguez-Esquerre, V. F., OY

Roedig, Utz, $1 T$

Rosová, A., $1 \mathrm{~K}$

Rubio-Mercedes, C. E., OY

Sabad-e-Gul, OC

Samaali, Hatem, OA

Sarangan, Andrew, 11

Savin, Adriana, $1 \mathrm{~N}$

Sekiguchi, Atsushi, IB

Sensale-Rodriguez, Berardi, IP

Sexton, James, $1 T$

Sharma, Manish, OF

Sheu, Fang-Wen, $1 \mathrm{M}$

Shokeir, Hamzah, $1 T$

Sojková, M., IK

Song, Byonggwon, 01

Song, Lixin, 1D

Stein, Ori, OL, $1 \mathrm{U}$

Streit, J., OL

Sugino, Naoto, 1B, 1E, 1F, 1Q

Sul, Soohwan, 01

Takei, Satoshi, 14, 1B, 1E, 1F, 1Q

Tandon, R. P., OV

Tomar, Monika, OS, OV

Turchenko, Vitalii, IN

Vergara, Pedro P., 04

Vincent, Grégory, 06

Wang, Xinbo, $1 \mathrm{P}$

Wei, Yu-Chien, 17

Weiss, Sharon M., 07

Werner, Thomas, 03

Woodhead, Christopher S., IT

Wu, Junjie, 12

Yan, Li, 1D

Yang, Henglong, 17

Yang, Kiyeon, 0 I

Yokogawa, Toshiya, 00

Young, Robert J., $1 \mathrm{~T}$

Zawawi, S. A., $1 \mathrm{~J}$

Zhang, Yi, 1D 


\section{Conference Committee}

Symposium Chairs

Harry A. Atwater Jr., California Institute of Technology (United States)

Nikolay I. Zheludev, Optoelectronics Research Centre (United

Kingdom) and Nanyang Technological University (Singapore)

Symposium Co-Chairs

James G. Grote, Air Force Research Laboratory (United States)

David L. Andrews, University of East Anglia (United Kingdom)

Conference Chairs

Eva M. Campo, Bangor University (United Kingdom)

Elizabeth A. Dobisz, Spin Transfer Technologies, Inc. (United States)

Louay A. Eldada, Quanergy Systems, Inc. (United States)

Conference Program Committee

André-Jean Attias, Université Pierre et Marie Curie (France)

Maziar Ghazinejad, California State University, Fresno (United States)

Sarah Haigh, The University of Manchester (United Kingdom)

Ghassan E. Jabbour, Arizona State University (United States)

Robert Magnusson, The University of Texas at Arlington (United States)

Balaji Panchapakesan, Worcester Polytechnic Institute (United States)

Won Park, University of Colorado at Boulder (United States)

Dorota A. Pawlak, Institute of Electronic Materials Technology (Poland)

Michael T. Postek, National Institute of Standards and Technology (United States)

Dianne L. Poster, National Institute of Standards and Technology (United States)

Anne E. Sakdinawat, SLAC National Accelerator Laboratory (United States)

Jun Tanida, Osaka University (Japan)

Richard Tiberio, Stanford University (United States)

Chee Wei Wong, Columbia University (United States)

Wei Wu, The University of Southern California (United States) 


\section{Session Chairs}

1 Photonic Devices

Elizabeth A. Dobisz, Spin Transfer Technologies, Inc. (United States)

2 Innovative Devices

Wen-Di Li, The University of Hong Kong (Hong Kong, China)

3 Nanopatterning

Elizabeth A. Dobisz, Spin Transfer Technologies, Inc. (United States)

4 Engineered Materials

Eva M. Campo, Bangor University (United Kingdom)

5 Carbon Nanotubes and Low-Dimensional Materials

Eva M. Campo, Bangor University (United States)

$6 \quad$ Light Trapping and Light Guiding

Balaji Panchapakesan, Worcester Polytechnic Institute (United States)

$7 \quad$ Nano-Optic Devices and Optic Engineering

Eva M. Campo, Bangor University (United Kingdom) 